\title{
IMMERSIONS OF REAL FLAG MANIFOLDS
}

\author{
R. E. STONG
}

\begin{abstract}
This paper shows that, in many cases, Lam's immersions of real flag manifolds are best possible.
\end{abstract}

\section{Introduction. In [2], K. Y. Lam proved}

THEOREM. The real flag manifold

$$
R F\left(n_{1}, n_{2}, \ldots, n_{s}\right)=O\left(n_{1}+n_{2}+\cdots+n_{s}\right) / O\left(n_{1}\right) \times O\left(n_{2}\right) \times \cdots \times O\left(n_{s}\right)
$$

can be immersed in Euclidean space with codimension $\frac{1}{2} \sum n_{i}\left(n_{i}-1\right)$, provided this codimension is nonzero.

The purpose of this note is to observe that this result is frequently best possible. Specifically, one has

Proposition. If $\omega=\left\{n_{1}, \ldots, n_{s}\right\}$ can be partitioned as $\omega=\omega_{1} \cup \omega_{2} \cup \omega_{3}$ where $\omega_{1}, \omega_{2} \neq \varnothing$,

(1) $\left|\sum_{n \in \omega_{1}} n-\sum_{n \in \omega_{2}} n\right| \leqslant 1$, and

(2) $m \in \omega_{3}$ implies $m \leqslant \Sigma_{n \in \omega_{1}} n+\sum_{n \in \omega_{2}} n+1$, then Lam's result is best possible for $R F(\omega)$.

For Grassmannians, $R F(p, q)$, this result was proved by Hiller and myself [1], and the hypothesis is simply that $q=p-1, p$, or $p+1$. In fact, the proof is a reduction to the Grassmannian case.

I am indebted to the National Science Foundation for financial support during this work.

2. The proof. According to the argument given by Lam [2, p. 313] the normal bundle of $R F\left(n_{1}, \ldots, n_{s}\right)$ is realized by

$$
\eta=\lambda^{2} \xi_{1} \oplus \lambda^{2} \xi_{2} \oplus \cdots \oplus \lambda^{2} \xi_{s},
$$

where $\xi_{i}$ is the standard $n_{i}$-plane bundle, and $\lambda^{2}$ denotes the second exterior power. The goal is to prove that the top-dimensional Stiefel-Whitney class of this bundle $\eta$ is nonzero.

LEMMA 1. If $\omega$ refines $\omega^{\prime}$ and if the top normal Stiefel-Whitney class of $R F\left(\omega^{\prime}\right)$ is nonzero, then the top normal Stiefel-Whitney class of $R F(\omega)$ is nonzero.

Received by the editors August 28, 1982.

1980 Mathematics Subject Classification. Primary 57R42. 
Proof. Inductively, it suffices to consider $\omega=\left\{n_{1}, n_{2}, \ldots, n_{s}\right\}$ and $\omega^{\prime}=\left\{n_{1}+\right.$ $\left.n_{2}, n_{3}, \ldots, n_{s}\right\}$ adding together two of the $n_{i}$. One then has a fibration $\pi: R F(\omega) \rightarrow$ $R F\left(\omega^{\prime}\right)$ with fiber $R F\left(n_{1}, n_{2}\right)$, with $\pi^{*}$ monic in $\bmod 2$ cohomology (since the cohomology injects into that of $R F(1, \ldots, 1))$. The map $\pi$ splits the $\left(n_{1}+n_{2}\right)$-plane bundle over $R F\left(\omega^{\prime}\right)$ into $\xi_{1} \oplus \xi_{2}$ and hence

$$
\pi^{*}\left(\eta_{\omega^{\prime}}\right)=\lambda^{2}\left(\xi_{1} \oplus \xi_{2}\right) \oplus \lambda^{2} \xi_{3} \oplus \cdots \oplus \lambda^{2} \xi_{s}=\left(\xi_{1} \otimes \xi_{2}\right) \oplus \eta_{\omega}
$$

Thus $w_{\text {top }}\left(\eta_{\omega}\right) \cdot w_{\text {top }}\left(\xi_{1} \otimes \xi_{2}\right)=\pi^{*} w_{\text {top }}\left(\eta_{\omega^{\prime}}\right) \neq 0$ and so $w_{\text {top }}\left(\eta_{\omega}\right) \neq 0$.

If one applies this lemma to the Proposition, adding together the integers in $\omega_{1}$ and the integers in $\omega_{2}$, one is reduced to consideration of $R F\left(p, q, m_{1}, \ldots, m_{t}\right)$ where $q=p-1, p$, or $p+1$ and each $m_{i} \leqslant p+q+1$.

Lemma 2. If $n_{1} \leqslant n_{2}+\cdots+n_{s}+1, s \geqslant 3$, and the top normal Stiefel-Whitney class of $R F\left(n_{2}, \ldots, n_{s}\right)$ is nonzero, then the top normal Stiefel-Whitney class of $R F\left(n_{1}, n_{2}, \ldots, n_{s}\right)$ is nonzero.

Proof. Consider the map $R F(1, \ldots, 1) \stackrel{\pi}{\rightarrow} R F\left(n_{1}, n_{2}, \ldots, n_{s}\right)$ so that

$$
w\left(\pi^{*} \xi_{1}\right)=\prod_{1}^{n_{1}}\left(1+e_{i}\right), \ldots, w\left(\pi^{*} \xi_{j}\right)=\prod_{1}^{n_{j}}\left(1+e_{n_{1}+\cdots+n_{j},+i}\right), \ldots
$$

writes each Stiefel-Whitney class in terms of one-dimensional classes. Here $R F(1, \ldots, 1)=\operatorname{Flag}\left(R^{n_{1}+\cdots+n_{s}}\right)$, for which the $\bmod 2$ cohomology was described in [1]. One then has

$$
\pi^{*} w_{\mathrm{top}}(\eta)=\prod_{i=1}^{s} s_{\left(n_{i}-1, \ldots, 1\right)}\left(e_{n_{1}+\cdots+n_{i-1}+1}, \ldots, e_{n_{1}+\cdots+n_{i, 1}+n_{i}}\right)
$$

by applying the lemma on p. 364 of [1]. Let $m=n_{2}+\cdots+n_{s}$ and

$$
a=e_{1}^{n_{1}+m-1} e_{2}^{n_{1}+m-3} \cdots e_{j}^{n_{1}+m-2 j+1} \cdots e_{n_{1}}^{n_{1}+m-2 n_{1}+1}
$$

Then

$$
a \cdot \pi^{*} w_{\mathrm{top}}(\eta)=e^{n_{1}+m-1} \cdots e_{n_{1}}^{n_{1}+m-n_{1}}\left[\prod_{i=2}^{s} s_{\left(n_{i}-1, \ldots, 1\right)}\left(e_{\left.n_{1}+\cdots+n_{t-1}+1, \ldots\right)}\right)\right],
$$

which is nonzero if the second factor is nonzero in $\operatorname{Flag}\left(R^{m}\right)$.

Applying this lemma to remove the integers $m_{i}$ from $\omega_{3}$, one reduces the Proposition to the case $R F(p, q)$ where $q=p-1, p$, or $p+1$. This in turn was proved in [1], to give the Proposition.

Comment. There are precisely four $\omega=\left\{n_{1}, \ldots, n_{s}\right\}$ having each $n_{i} \leqslant 4$ to which the Proposition does not apply. They are $\{3,1\},\{4,1\},\{4,2\}$ and $\{4,1,1\}$, and each has an improved immersion. $R P^{3}$ immerses in $R^{4}, R P^{4}$ in $R^{7}, R F(4,2)$ is an oriented 8-dimensional manifold with $\bar{w}_{2} \bar{w}_{6}=0$ and immerses in $R^{14}$ by [3, Theorem (4.2.1)], while $R F(4,1,1) \subset R P^{5} \times R P^{5}$ immerses in $R^{7} \times R^{7}=R^{14}$. These improved immersions are best possible, since $\bar{w}_{3}, \bar{w}_{6}$ and $\bar{w}_{5}$ are nonzero for the three latter cases. 


\section{REFERENCES}

1. H. Hiller and R. E. Stong, Immersion dimension for real Grassmannians, Math. Ann. 255 (1981). 361-367.

2. K. Y. Lam, A formula for the tangent bundle of flag manifolds and related manifolds, Trans. Amer. Math. Soc. 213 (1975), 305-314.

3. M. E. Mahowald and F. P. Peterson, Secondary cohomology opertions on the Thom class, Topology 2 (1964), 367-377.

Department of Mathematics, University of Virginia, Charlottesville, Virginia 22903-3199 